\title{
Mémoire olfactive et migration neuronale chez l'adulte
}

\author{
Pierre-Marie Lledo, Alan Carleton, David Desmaisons, \\ Paul-Antoine Salin, Jean-Didier Vincent
}

L'odorat plus que tout autre sens a partie liée avec la mémoire. Il suffit d'évoquer à ce propos le rôle des souvenirs olfactifs dans la genèse des comportements fondamentaux de l'espèce comme le sexe ou l'alimentation, et de rappeler que les structures nerveuses qualifiées de "rhinencéphaliques» sont au cœur des processus mnésiques dans le cerveau. Le système olfactif bénéficie d'un statut particulier, en l'occurrence la capacité de

\begin{abstract}
produire des neurones pendant toute la vie de l'individu, y compris à l'âge adulte. Cette neurogenèse continue pose la question de savoir comment, dans un système dynamique soumis à un renouvellement permanent, sont conservés les différents aspects de la mémoire olfactive. L'examen des singularités du système olfactif permet d'ébaucher une réponse dans le même temps qu'il éclaire les relations entre mémoire et neurogenèse cérébrale.
\end{abstract}

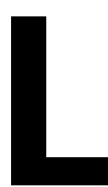

a première particularité du système olfactif vient de sa relative simplicité. Alors qu'en règle générale toute voie sensorielle comporte trois neurones, deux synapses seulement relient les neurones récepteurs aux neurones du cortex cérébral olfactif (ou paléocortex). Ce dernier est notablement moins complexe que le néocortex : trois couches cellulaires seulement, une structuration verticale rigoureuse et un réseau dense de fibres d'association dans le plan horizontal. Le bulbe olfactif, interposé entre les neurones sensoriels olfactifs et le cortex cérébral, possède une organisation comparable à celle du cortex olfactif. Comme ce dernier, il est la cible de nombreuses voies afférentes, dites centrifuges, et apparaît comme une véritable structure centrale. Premier relais de l'information olfactive, ses fonctions sont en fait plus étendues que la simple transmission des messages communiqués par les récepteurs olfactifs $[1,2]$.

\section{Le bulbe olfactif pourrait être le siège d'un stockage de I'information sensorielle}

En réponse à une stimulation olfactive, le bulbe présente une signature électrique traduisant l'activité synchronisée de neurones. Cette signature acquiert, lors d'un apprentissage, une organisation spatio-temporelle spécifique de l'odeur apprise qui se maintient durablement. Le bulbe olfactif pourrait donc conserver une trace des messages représentant des stimulus «signifiants» reçus dans une situation de forte motivation et d'éveil intense, autrement dit propice à l'apprentissage. Ainsi, chez un raton exposé à une odeur pendant qu'une stimulation tactile simule le léchage par la mère, le bulbe olfactif présente, au niveau des régions activées par cette même odeur, des changements métaboliques et structuraux. Dans le même temps, le raton acquiert une préférence pour cette odeur. La suppression des fibres noradrénergiques dans le bulbe empêche cet apprentissage et la traduction électrique de la trace bulbaire correspondante. De même, la souris femelle n'est plus capable de garder une empreinte olfactive du mâle qui vient de la féconder. La présence de ce dernier induit alors un avortement, au même titre que celle d'un partenaire sexuel étranger [1] (effet Bruce-Whitten). Dans ce dernier cas, il a été vérifié que seuls les aspects relatifs à la mémoire sont perturbés et non la capacité perceptive elle-même. Ces connexions centrifuges ont pour principale cible la population la plus nombreuse des neurones du bulbe olfactif: les cellules granulaires. Ces interneurones inhibiteurs modulent l'activité des cellules principales (cellules mitrales et à panache) et jouent un rôle fondamental dans l'apprentissage olfactif (pour revue, voir [1]). 


\section{Le bulbe olfactif est le siège d'une neurogenèse permanente}

Fait exceptionnel dans le système nerveux central de l'adulte, le système olfactif conserve une capacité de neurogenèse tant au niveau des entrées (cellules sensorielles) qu'au niveau des interneurones bulbaires. Si la régénération des neurorécepteurs localisés dans la muqueuse olfactive peut s'expliquer par la nécessité d'un remplacement des neurones détruits par les agressions du monde extérieur (virus, agents chimiques, etc.), la raison du renouvellement des interneurones situés dans les couches plus profondes du bulbe olfactif reste énigmatique. En effet, des cellules de la zone subventriculaire des ventricules latéraux continuent de proliférer chez l'adulte pour donner naissance aux interneurones du bulbe olfactif. Une longue migration conduit ces cellules en formation jusqu'aux couches granulaire et glomérulaire du bulbe olfactif homolatéral $[3,4]$ où elles se différencient respectivement en cellules granulaires et périglomérulaires, les deux catégories d'interneurones bulbaires.

Nous avançons l'hypothèse selon laquelle cette neurogenèse pourrait être le support d'une forme de mémoire similaire à celle décrite dans le cas de l'apprentissage du chant chez l'oiseau [5]. Contrairement aux cellules germinales de la zone ventriculaire qui s'organisent radialement [6], les cellules de la zone subventriculaire ne présentent aucune organisation apparente [7]. Si, dans la zone ventriculaire, les divisions cellulaires prennent fin avant la naissance, la prolifération dans la zone subventriculaire persiste jusqu'à la vie adulte $[7,8]$. Des études réalisées in vitro ont démontré que les cellules germinales de la zone subventriculaire peuvent se différencier en neurones lorsqu'elles sont isolées à partir d'animaux adultes $[8,9]$. Les injections systémiques de thymidine tritiée ont montré qu'une de leurs principales destinations est le bulbe olfactif $[10,11]$, mais leur devenir n'était pas connu. Ainsi, des travaux ont indiqué que les cellules ger- minales de la zone subventriculaire meurent rapidement après leur passage en mitose [12] ou deviennent des cellules gliales [13-19].

Ce n'est que très récemment, en suivant le trajet de petits amas cellulaires provenant de la zone subventriculaire dans le cerveau de rongeurs nouveaunés [3] ou adultes [4], que la preuve d'un flux migratoire vers le bulbe olfactif, suivi d'une différenciation en neurones, a été rapportée. Grâce à l'injection de différents traceurs dans la zone subventriculaire antérieure (thymidine tritiée, marqueurs fluorescents, rétrovirus ou transplantation de cellules marquées génétiquement), il a été possible d'identifier le bulbe olfactif comme destination unique des neurones en migration. Cette migration emprunte un trajet nommé courant de migration rostral [10]. Il s'étend le long des bords dorsal et rostral de la corne antérieure du ventricule latéral pour finir au cœur même du bulbe olfactif (figure 1). Ces études de marquage ont indiqué qu'une grande majorité des cellules migratrices donnent naissance aux cellules granulaires $(70 \%$ à $80 \%)$, les autres produisant des interneurones périglomérulaires et des cellules gliales.

Les précurseurs neuronaux forment des chaînes orientées selon l'axe longitudinal du ventricule latéral et sont capables de migration sur un plan tangentiel [20]. Chez la souris, la distance parcourue par les neuroblastes avant d'atteindre leur cible est d'environ $8 \mathrm{~mm}$. Si le nombre de cellules en cours de migration n'est pas encore connu, on sait, en revanche, qu'en quelques heures, 10000 à 20000 cellules se joignent au courant de migration rostrale [4]. La vitesse moyenne de migration y est de $30 \mu \mathrm{m} / \mathrm{h}$, c'est-à-dire quatre à cinq fois plus rapide que la migration radiale du cortex cérébral $[4,6]$. Autre particularité, ces neuroblastes possèdent la capacité de se diviser durant leur déplacement [21, 22], alors même que certaines protéines caractéristiques des neurones sont déjà synthétisées [23].

L'ensemble de ces propriétés - déplacement continu des cellules en grand nombre, vitesse élevée et division cellulaire pendant la migration - soulignent les particularités de cette migration dite "en chaîne».

\section{La migration en chaîne}

Les cellules emportées dans le courant migratoire rostral sont caractérisées par une morphologie allongée et la présence d'un prolongement antérieur qui se termine par un cône de croissance, comparable à celui trouvé à l'extrémité d'un axone en croissance. Ce dernier pourrait jouer

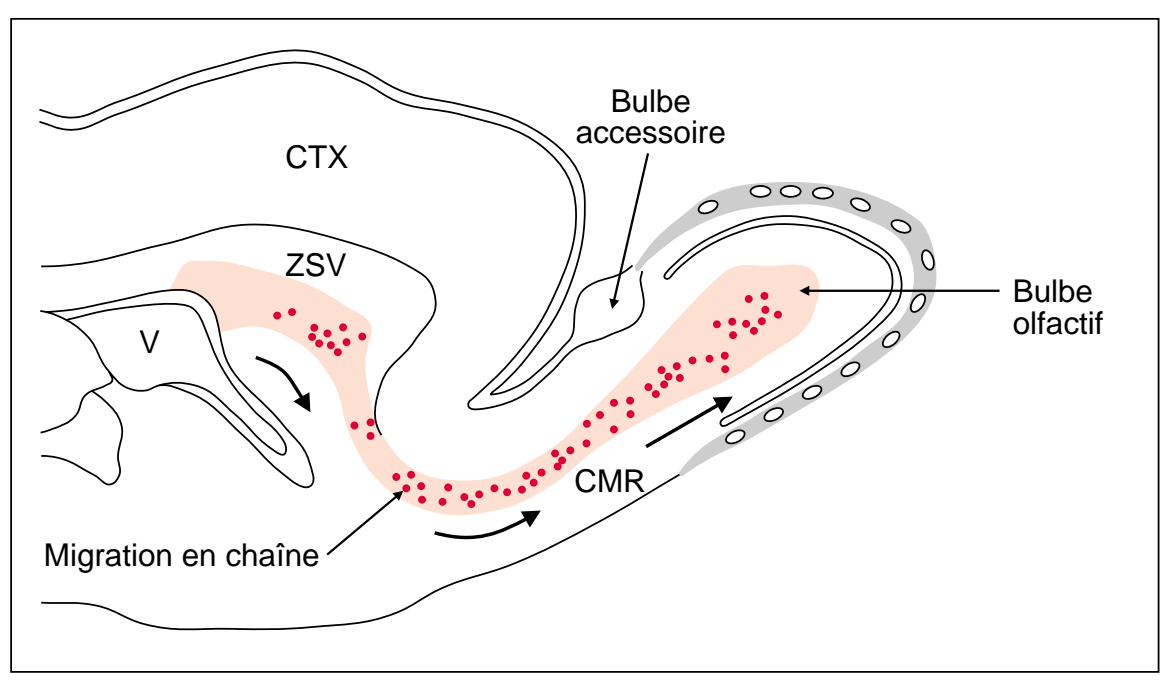

Figure 1. Coupe sagittale du cerveau de rat adulte. Des cellules de la zone subventriculaire (ZSV) continuent de proliférer chez l'adulte pour migrer vers le bulbe olfactif. Cette migration suit une voie appelée courant de migration rostral (CMR). V: ventricule; CTX: cortex. 
un rôle important dans le déplacement des neurones.

L'existence de protéines dites morphorégulatrices qui régissent l'adhérence et le déplacement des cellules au cours du développement est bien connue [25]. Leur principale fonction est de contrôler l'adhérence des cellules à un substrat et de relier les cellules entre elles. Les molécules d'adhérence neuronale (N-CAM) qui participent à la cohésion cellulaire par un mécanisme de liaisons homophiles, ont été particulièrement étudiées. Au cours du développement, ces molécules passent d'une forme embryonnaire riche en acide polysialique (PSA-N-CAM) à une forme adulte pauvre en PSA. Cette conversion de nature biochimique s'accompagne d'une perte de la capacité migratoire des cellules au profit d'une plus grande adhérence [26]. La forme embryonnaire PSA-N-CAM, caractéristique des structures en cours de développement, est toutefois encore exprimée dans les cerveaux adultes au niveau de sites pouvant subir de profonds remaniements morpho-fonctionnels [27, 28]. Des travaux centrés autour de cette forme ont fourni d'importants indices sur les mécanismes migratoires des précurseurs neuronaux du bulbe olfactif et révélé une forte expression de PSAN-CAM à la surface de cellules du courant migratoire [29] qui sont organisées sous forme de longs cordons [21, 27, 30]. L'invalidation partielle (un exon de $180 \mathrm{~kb}$ ) [31], ou totale du gène $N-C A M$ [32], s'accompagne d'une importante réduction de la migration des précurseurs neuronaux du bulbe olfactif. Le développement général du cerveau reste normal, mais une accumulation de cellules dans la zone subventriculaire et une atrophie bulbaire témoignent d'une interruption plus ou moins complète du flux [33]. Des expériences de transplantations tissulaires ont indiqué par ailleurs que les défauts de migration observés après suppression, par manipulation génétique du gène $N-C A M$, chez la souris, résultent bien de l'absence de la molécule d'adhérence dans le courant migratoire [34]. L'ensemble de ces résultats souligne l'importance des résidus PSA dans la migration tan- gentielle des précurseurs neuronaux du bulbe olfactif.

Le courant migratoire comprend deux types cellulaires $[29,35]$ : les cellules de type A qui correspondraient aux neuroblastes en migration et les cellules de type $\mathrm{B}$, riches en protéines gliales fibrillaires (GFAP), qui seraient des astrocytes. Les coupes sériées du courant de migration ont mis en évidence un agencement topographique particulier. Les chaînes sont uniquement formées des corps cellulaires et des prolongements des neuroblastes. Les astrocytes qui possèdent de multiples prolongements orientés selon la direction de migration forment une gaine tubulaire qui enveloppe les neuroblastes. Pendant la migration, les jeunes neurones se déplaceraient donc ensemble, les uns contre les autres, dans un tunnel formé par les cellules gliales. Les cellules en migration établiraient des contacts très étroits entre elles, mais jamais avec les cellules gliales environnantes.

Cette migration en chaîne décrite chez l'adulte représente une nouvelle forme de déplacement de précurseurs neuronaux fondée sur des interactions homotypiques ne faisant pas appel aux «rails» axonaux ou gliaux (caractéristiques du cerveau embryonnaire). Des cellules du cortex cérébral [36] ou du cervelet [29] qui migrent normalement le long de prolongements gliaux, lorsqu'elles sont greffées dans la zone subventriculaire antérieure, perdent leur capacité de migration. Ce résultat conforte l'idée selon laquelle la migration en chaîne serait une forme particulière de déplacement neuronal propre à certains types cellulaires. Il indique également que les signaux cellulaires de surface utilisés lors de la migration radiale sont différents de ceux utilisés pour la migration en chaîne.

La majorité des précurseurs neuronaux du courant migratoire rostral sont orientés vers le bulbe olfactif. Cette observation est en faveur de la présence, le long du trajet, d'indicateurs de direction. Cette direction pourrait résulter d'une polarisation endogène des précurseurs neuronaux ou dépendre de gradients chimiques (attractifs ou répulsifs).
Cependant, il est vraisemblable que le bulbe olfactif ne sécrète aucune de ces molécules, les cellules de la zone subventriculaire n'étant pas sensibles à la présence in vitro d'un explant du bulbe olfactif [37] ou à la destruction in vivo du bulbe olfactif. En revanche, une activité chémo-répulsive a été mise en évidence au niveau du septum caudal [37]. Les cellules de la zone subventriculaire, placées auprès d'un explant de septum caudal, migrent de façon asymétrique: elles ont pour point de départ le côté opposé à l'explant de septum.

La fonction exacte de la gaine gliale qui entoure les chaînes de cellules en cours de migration reste à découvrir. Elle pourrait former une simple barrière mécanique qui empêche l'échappée des neurones migrateurs en dehors du courant ou bien priver les cellules de tout contact avec des facteurs présents dans le parenchyme cérébral environnant. Cette gaine enfin pourrait fournir des substances importantes pour la survie, la différenciation ou l'orientation des neuroblastes en cours de migration. Cette nouvelle forme de migration serait utilisée dans le cerveau adulte pour les déplacements rapides, empruntant une voie tangentielle et nécessitant l'apport d'un nombre élevé de cellules. Les mécanismes impliqués dans cette migration tangentielle, tant au niveau embryonnaire $[38,39]$ que chez l'adulte [29, 35], ne sont pas encore connus. Définir la nature des interactions entre les jeunes neurones en déplacement, ainsi que le rôle précis de PSA-N-CAM devrait permettre de mieux appréhender la fonction de cette migration particulière.

Le système nerveux central adulte est plus accessible que le cerveau embryonnaire aux manipulations expérimentales et la production neuronale s'y effectue sur une plus longue durée. Ces caractéristiques rendent le modèle du bulbe olfactif intéressant pour l'étude des mécanismes de migration cellulaire, de guidage et de différenciation neuronale chez l'adulte. De nombreuses questions restent en suspens: comment une telle migration est-elle établie et maintenue sur une aussi longue durée? Quelle est la nature et la source de la population de cellules 
souches qui permet une production continuelle d'interneurones dans le cerveau adulte? Pourquoi le remplacement d'interneurones du bulbe olfactif est-il nécessaire au niveau des cerveaux jeunes et adultes? Existe-t-il des facteurs capables de déclencher ou de régler cette migration en chaîne?

\section{Hypothèse}

A chaque entrée d'air, une «carte d'activité olfactive» s'inscrit à l'entrée du bulbe et vient interagir avec l'activité spontanée des neurones bulbaires. Cette carte, d'abord glomérulaire (figure 2), est traitée par les interneurones périglomérulaires qui en déterminent les contours grâce à une action inhibitrice de voisinage [40]. Elle est ensuite transférée vers les couches plus profondes par l'intermédiaire des dendrites primaires des cellules mitrales. L'excitation des cellules granulaires induit une inhibition en retour des cellules mitrales, amorçant ainsi une oscillation de l'ensemble du bulbe. Celle-ci se traduit sur l'électroencéphalogramme par un motif d'ondes modulées spatialement dans leur amplitude et leur phase. La carte d'activité imposée à la couche glomérulaire d'entrée donne donc naissance à un motif plurineuronal de sortie, dirigé vers les aires paléocorticales. Les variations de la population des cellules granulaires, par l'intermédiaire d'une neurogenèse accompagnée d'une migration continue, pourraient moduler ces cartes de sortie. Les recherches actuelles s'efforcent d'évaluer le degré de plasticité de ces circuits et de déterminer les facteurs qui influent sur leur fonctionnement. Le bulbe pourrait être le siège d'un premier traitement du message olfactif, qui serait ensuite transféré vers d'autres régions cérébrales.

Une meilleure compréhension de la dynamique neuronale du système olfactif devrait permettre d'élargir les champs d'investigation centrés sur la neurobiologie de la mémoire et de l'apprentissage qui jusqu'alors restaient limités au cadre de la synapse. Le paradigme actuel place une modification de l'efficacité synaptique à

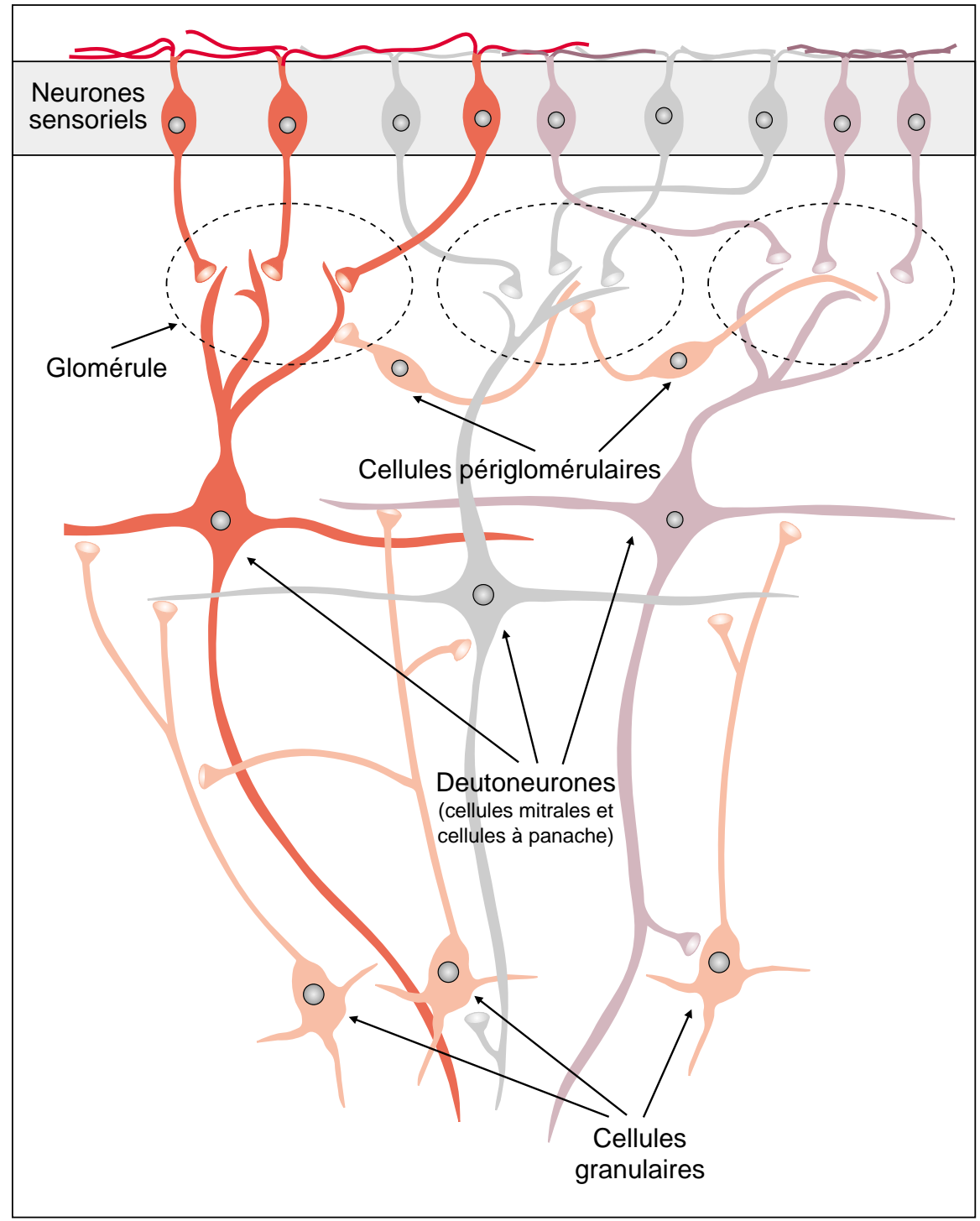

Figure 2. Représentation schématique des connexions intrabulbaires. Les cellules réceptrices (neurones sensoriels) de la muqueuse olfactive envoient leurs axones à l'entrée du bulbe olfactif, à travers la lame criblée de l'os ethmoïde, dans les glomérules où ils contactent les dendrites primaires des cellules mitrales. Ces neurones, de projection, sont contrôlés par deux catégories de neurones locaux inhibiteurs, les cellules périglomérulaires et les cellules granulaires.

mémoire ( $\mathrm{m} / \mathrm{s} n^{\circ} 5$, vol. 13, p. 698). Selon cette hypothèse, la mise en place de véritables engrammes, dans un réseau neuronal donné, suppose une réorganisation anatomique et fonctionnelle des connexions entre neurones. Ce remaniement s'effectuerait selon un processus de sélection qui exclurait toute participation de phénomènes constructifs. L'étude du système olfactif devrait permettre de replacer les remaniements mor- pho-fonctionnels observés chez l'adulte dans le domaine plus vaste de celui de l'épigenèse qui régit le développement $\mathrm{d}$ 'un système nerveux central en construction permanente (pour revue, voir [41])

Remerciements

Nous remercions très vivement le Dr Alain Prochiantz pour ses critiques et ses conseils.

$m / s n^{\circ} 6-7$, vol. 14 , juin-juillet 98 


\section{RÉFÉRENCES}

1. Keverne EB. Olfactory learning. Curr Opin Neurobiol 1995 ; 5 : 482-8.

2. Parmentier M, Vanderhaegen P, Schurmans S, Libert F, Vassart G. Génétique moléculaire des récepteurs olfactifs. $\mathrm{Med}$ Sci 1994; 10 : 1083-90.

3. Luskin MB. Restricted proliferation and migration of postnatally generated neurons derived from the forebrain subventricular zone. Neuron 1993; 11: 173-89.

4. Lois C, Alvarez-Buylla A. Long-distance neuronal migration in the adult mammalian brain. Science 1994; 264 : 1145-8.

5. Alvarez-Buylla A, Kim JR, Nottebohm F. Birth of projection neurons in adult avian brain may be related to perceptual or motor learning. Science 1990; 249 : 1444-6.

6. Jacobson M. Developmental neurobiology. New York: Plenum Press, 1991.

7. Smart I. The subependymal layer of the mouse-brain and its cell production as shown by radioautography after thymidine-H3 injection. J Comp Neurol 1961; 116: 325-48.

8. Lois C, Alvarez-Buylla A. Proliferating subventricular zone cells in the adult mammalian forebrain can differentiate into neurons and glia. Proc Natl Acad Sci USA 1993; 90: 2074-7.

9. Kirschenbaum B, Goldman SA. Brainderived neurotrophic factor promotes the survival of neurons arising from the adult rat forebrain subependymal zone. Proc Natl Acad Sci USA 1995; 92 : 210-4.

10. Altman J. Autoradiographic and histological studies of postnatal neurogenesis. IV. Cell proliferation and migration in the anterior forebrain, with special reference to persisting neurogenesis in the olfactory bulb. J Comp Neurol 1969; 137 : 433-58.

11. Shimada M. Cytokenetics and histogenesis of early postnatal mouse brain as studied by $3 \mathrm{H}$-thymidine autoradiography. Arch Histol Jap 1966; 26 : 413-37.

12. Morshead CM, Van der Kooy D. Postmitotic death is the fate of constitutively proliferating cells in the subependymal layer of the adult mouse brain. I Neurosci 1992; 12 : 249-56.

13. Smart I, Leblond CP. Evidence for division and transformations of neuroglia cells in the mouse brain, as derived from radioautography after injection of thymidine-H3 . J Comp Neurol 1961; 116: 349-67.

14. Paterson JA, Privat A, Ling EA, Leblond CP. Investigation of glial cells in semithin sections. III. Transformation of subependymal cells into glial cells, as shown by radioautography after ${ }^{3} \mathrm{H}$-thymidine injection into the lateral ventricle of the brain of young rats. J Comp Neurol 1973; 149: 83-102.

15. Privat A, Leblond CP. The subependymal layer and neighboring region in the

$m / s n^{\circ} 6-7$, vol. 14 , juin-juillet 98 brain of the young rat. J Comp Neurol 1972; 146: 277-302.

16. Levison SW, Chuang C, Abramson BJ, Goldman JE. The migrational patterns and developmental fates of glial precursors in the rat subventricular zone are temporally regulated. Development 1993 ; 119:611-22.

17. Goldman JE. Lineage, migration, and fate determination of postnatal subventricular zone cells in the mammalian CNS. J Neurooncol 1995 ; 24 : 61-4.

18. Levison SW, Goldman JE. Both oligodendrocytes and astrocytes develop from progenitors in the subventricular zone of postnatal rat forebrain. Neuron 1993; 10: 201-12.

19. Zerlin M, Levison SW, Goldman JE. Early patterns of migration, morphogenesis, and intermediate filament expression of subventricular zone cells in the postnatal rat forebrain. J Neurosci 1995 ; 15 : 7238-49.

20. Doetsch F, Alvarez-Buylla A. Network of tangential pathways for neuronal migration in adult mammalian brain. Proc Natl Acad Sci USA 1996; 93: 14895-900.

21. Rousselot P, Lois C, Alvarez-Buylla A. Embryonic (PSA) N-CAM reveals chains of migrating neuroblasts between the lateral ventricle and the olfactory bulb of adult mice. J Comp Neurol 1995; 351 : 51-61.

22. Luskin MB, Boone MS. Rate and pattern of migration of lineally-related olfactory bulb interneurons generated postnatally in the subventricular zone of the rat. Chem Senses 1994; 19: 695-714.

23. Menezes JRL, Smith CM, Nelson KC, Luskin MB. The division of neuronal progenitor cells during migration in the neonatal mammalian forebrain. Mol Cell Neurosci $1995 ; 6: 496-508$.

24. Kishi K. Golgi studies on the development of granule cells of the rat olfactory bulb with reference to migration in the subependymal layer. I Comp Neurol 1987; 258: $112-24$.

25. Edelman GM, Rutishauser U. Molecules involved in cell-cell adhesion during development. J Supramol Struct Cell Biochem 1981; 16: 259-68.

26. Rutishauser U, Watanabe M, Silver J, Troy FA, Vimr ER. Specific alteration of NCAM-mediated cell adhesion by an endoneuraminidase. J Cell Biol 1985; 101 : 1842-9.

27. Seki T, Arai Y. Distribution and possible roles of the highly polysialylated neural cell adhesion molecule (NCAM-H) in the developing and adult central nervous system. Neurosci Res 1993; 17: 265-90.

28. Bonfanti L, Olive S, Poulain DA, Theodosis DT. Mapping of the distribution of polysialylated neural cell adhesion molecule throughout the central nervous system of the adult rat: an immunohistochemical study. Neuroscience 1992; 49: 419-36.
29. Jankovski A, Sotelo C. Subventricular zone-olfactory bulb migratory pathway in the adult mouse: cellular composition and specificity as determined by heterochronic and heterotopic transplantation. J Comp Neurol 1996 ; 371 : 376-96.

30. Bonfanti L, Theodosis DT. Expression of polysialylated neural cell adhesion molecule by proliferating cells in the subependymal layer of the adult rat in its rostral extensive and in the olfactory bulb. Neuroscience 1994; 62 : 291-305.

31. Tomasiewicz H, Ono K, Yee D, Thompson C, Goridis C, Rutishauser U, Magnuson T. Genetic deletion of a neural cell adhesion molecule variant (N-CAM 180) produces distinct defects in the central nervous system. Neuron 1993; 11 : 1163-74.

32. Cremer H, Lange R, Christoph A, Plomann M, Vopper G, Roes I, Brown R, Baldwin S, Kraemer P, Scheff S, Barthels D, Rajewsky K, Wille W. Inactivation of the NCAM gene in mice results in size reduction of the olfactory bulb and deficits in spatial learning. Nature 1994; 367 : 455-9.

33. Ono K, Tomasiewicz $\mathrm{H}$, Magnuson $\mathrm{T}$, Rutishauser U. N-CAM mutation inhibits tangential neuronal migration and is phenocopied by enzymatic removal of polysialic acid. Neuron 1994; 13: 595-609.

34. $\mathrm{Hu} \mathrm{H}$, Tomasiewicz $\mathrm{H}$, Magnuson $\mathrm{T}$, Rutishauser $\mathrm{U}$. The role of polysialic acid in migration of olfactory bulb interneuron precursors in the subventricular zone. Neuron 1996; 16: 735-43.

35. Lois C, Garcia-Verdugo JM, AlvarezBuylla A. Chain migration of neuronal precursors. Science 1996; 271 : 978-81.

36. Zigova T, Betarbet R, Soteres BJ, Brock $\mathrm{S}$, Bakay RAE, Luskin MB. A comparison of the patterns of migration and the destinations of homotopically transplanted neonatal subventricular zone cells and heterotopically transplanted telencephalic ventricular zone cells. Dev Biol 1996; 173: 1-16.

37. Hu H, Rutishauser U. A septum-derived chemorepulsive factor for migrating olfactory interneuron precursors. Neuron 1996; $16: 933-40$

38. O'Rourke NA, Sullivan DP, Kaznowski CE, Jacobs AA, McConnell SK. Tangential migration of neurons in the developing cerebral cortex. Development 1995; 121 : 2165-76.

39. Walsh C, Cepko CL. Widespread dispersion of neuronal clones across functional regions of the cerebral cortex. Science 1992; 255: 434-40.

40. Sheperd G. Discrimination of molecular signals by the olfactory receptor neuron. Neuron 1994; 13: 771-90.

41. Doupe AJ. Songbirds and adult neurogenesis: a new role for hormones. Proc Natl Acad Sci USA 1994; 91 : 7836-8. 


\section{Pierre-Marie Lledo}

Chargé de recherche au Cnrs.

\section{Alan Carleton \\ Doctorant, Université Paris XI. \\ David Desmaisons \\ Doctorant, Université Paris VI. \\ Paul-Antoine Salin \\ Chargé de recherche au Cnrs.}

\section{Jean-Didier Vincent}

Professeur à la Faculté de médecine ParisSud, Université Paris XI, Institut universitaire de France.

Institut Alfred-Fessard, Cnrs, avenue de la Terrasse, 91198 Gif-sur-Yvette Cedex, France.

\section{Summary}

Olfactory memory

and neuronal migration in the adult

Neuroblasts in the subventricular zone of the walls of the lateral ventricle in the brain of young and adult rodents migrate into the olfactory bulb where they differentiate into local inter-neurons. These cells move closely associated with each other, forming chains without radial glial or axonal guidance. The migrating neuroblasts express PSA-NCAM on their surface and PSA residues are crucial for cell-cell interaction during chain migration. Cells remain organized as a chain formed by homotypic interactions between cells until they reach the olfactory bulb, where they disperse radially as individual neurons. We propose that a combination of neurogenesis and neuronal replacement in the olfactory system provides unique advantages for olfactory learning.

\section{TIRÉS À PART}

Alsace-France, 25-28 septembre / September 25-28

Cascades de prolifération et proto-oncogènes Proliferation cascades and protooncogenes

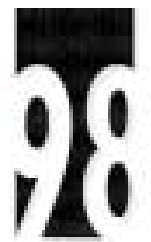

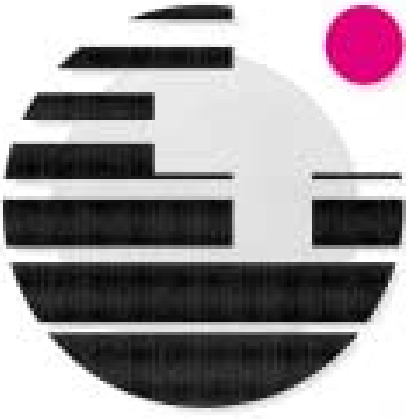

Hormones et Régulation Cellulaire XXIII" Symposium Européen du Mont $\mathbf{S}^{\mathrm{w}}$-Odile

Hormones and Cell Regulation XXIIIrd European Symposium of Mont $S^{\text {tw}}$-Odile

IWSCKPTDAN SOT SOUHSICN OES ASSLACS AEgistsanow ano suswistow of ABgracts

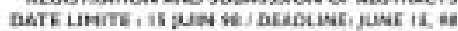

infoamation $6 \mathrm{~T}$ insckathon

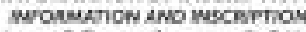

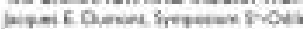

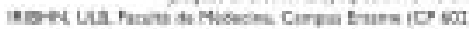

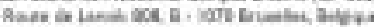

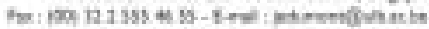

Récepteurs et cascades prolliératives Receptors and proliferative coscodes

Protéines kinases Protein kinases Transeription foctors

Cyclines CDK, Rb CDK, Rb cyelins

avte tarot on suspoarrn of
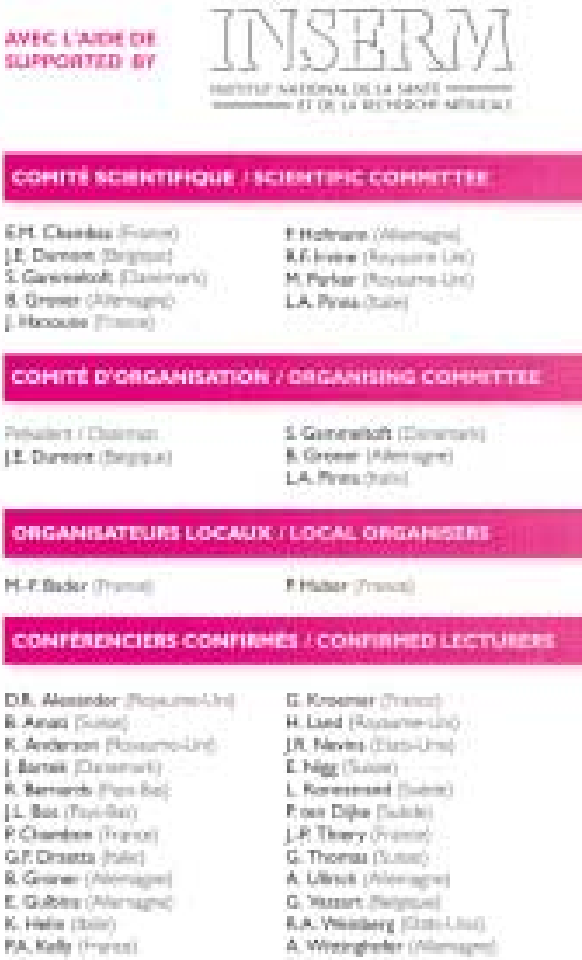

Facteurs de transcription

\section{CONFÉRENCES JACQUES MONOD 1998}

INTERACTIONS ENTRE LES PARASITES ET LE SYSTĖME IMMUNITAIRE : Protection ou paihologie?

$$
\text { AUSSOIS (France) - 14-18 septembre } 1998
$$

Président: $\quad$ WILSON R. Alan

University of York, Department of Biology, P.O. Box 373, UK-York YO1 5YW, United Kingdom Phone-Téléphone : + 441904432830 - Fax-Télécopie : + 441904432884

E-mail - Courtier électronique : raw3@york.ac.uk

Conférenciers : Barrel-Netto M., Behr C., Buzoni-Gatel D., Capron A., Capron M., Correa-Oliveira R., Dessein A. Dobbelaere D., Druilhe P., Dubremetz J.F., Finkelman F., Grau G., Grencis R., Grimaud J.-A., Gross U., Hill A., Hoffman S., Hontebeyrie M., Kaslow D., Langhorne J., Lovis J., Maizels R., Miller H., Milon G., Nutman T., Puijalon O., Riley E., Scott P., Sher A., Wilson R.A. 